\title{
Teaching shape bias to increase the expressive vocabularies of late talkers
}

\author{
Hannah Niese, Tim Brackenbury \\ Communication Sciences and Disorders, Bowling Green State University, Bowling Green, USA
}

Purpose: Shape bias is a lexical principle that suggests shape is the primary feature by which concrete noun labels apply to other objects. It helps children generalize the labels of newly encountered words to other referents. Shape bias typically develops around two years of age and has been linked with increases in expressive vocabulary. Smith et al. [1], for example, found significant increases in 17-month-olds' noun vocabularies following an intervention that taught shape bias.

Methods: The present investigation, a non-randomized pilot study, applied an eight-week shape bias-based intervention to Late Talkers who were between the ages of 23 and 34 months. The eight participants were exposed to novel objects, associated nonsense spoken labels, and objects that did and did not match their shape. Pre- and post-treatment expressive vocabularies were measured using the MacArthur-Bates Communicative Development Inventory [2].

Results: The participants increased their expressive vocabularies by 24 to 119 words, adding more new nouns than other word types.

Conclusions: These results, along with lessons learned about implementing the treatment, support larger-scale research on teaching shape bias to positively influence the expressive vocabularies of Late Talkers.

Keywords: Language impairment, Toddlers, Word learning, Statistical learning

\section{INTRODUCTION}

By the age of two, children's expressive semantic skills are rapidly expanding. These highly skilled word learners typically produce 200-300 different words, combine words into phrases, and use a variety of word types, such as nouns, verbs, adjectives, pronouns, and location words [3]. Not all two-year-olds, however, master these skills at the same rate. The term Late Talkers (LTs) describes children between the ages of 18 and 36 months who, a) have receptive language abilities that are generally age appropriate, $b$ ) do not have any obvious sensory, cognitive, or neurological deficits, but c) produce fewer words and word combinations than expected for their chronological age [4]. The criteria used for defining the low expressive vocabulary skills of LTs are typically cutoff scores based on the child's expressive vocabulary size (e.g., 50 words or less for a 24-month-old) or standard scores on expressive vocabulary measures (e.g., below the 10th percentile). Successful interventions for LTs have primarily focused on increasing vocabulary exposure, providing contextual supports, and directly teaching target vo-

(
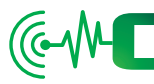

Received: June 10, 2020

Revision: September 4, 2020

Accepted: September 6, 2020

Correspondence:

Tim Brackenbury

Communication Sciences and Disorders, Bowling Green State University, Bowling Green, $\mathrm{OH} 43403$, USA

Tel: +419-372-2515

Fax: +419-372-8089

E-mail: tbracke@bgsu.edu

(C) 2020 The Korean Association of SpeechLanguage Pathologists

This is an Open Access article distributed under the terms of the Creative Commons Attribution NonCommercial License (https://creativecommons.org/ licenses/by-nc/4.0/) which permits unrestricted noncommercial use, distribution, and reproduction in any medium, provided the original work is properly cited. 
cabulary items [5]. Recent work by Alt and colleagues [6,7] suggest that interventions targeting cognitive skills that underlie word learning can also be effective. The present study builds on this work by exploring the feasibility of an intervention for LTs that focuses on the statistical learning-based lexical principle of shape bias.

\section{Late talkers}

A number of labels have been applied over the years to LTs, including children with slow expressive language development [8] and late language emergence [9]. Large scale prospective studies suggest that $13 \%$ to $20 \%$ of two-year-olds fit the definition of LTs $[9,10]$. Because vocabulary levels predict children's early grammatical development, LTs are at risk for later language problems [3]. Longitudinal group studies of LTs have revealed expressive semantic, morphosyntactic, and narrative skills that are below those of age matched control groups, but generally within or close to the average range [1113]. In addition to these group comparisons, outcome studies of LTs have revealed initial receptive-expressive vocabulary, receptive language, and socioeconomic status to be significant predictors of language outcomes [14]. Two common patterns of later language performance have been identified across the research on LTs. Between 50-75\% of two-year-olds identified as LTs perform within the average range on language measures as soon as one year later (Aka Late Bloomers) $[10,15]$. The remaining LTs continue to display identifiable expressive language impairments, including specific language impairment.

Approaches for advancing the expressive language skills of LTs are similar to those for toddlers with other language disorders. They include general language stimulation, focused language stimulation, and Milieu teaching [16]. Many, but not all, of the interventions also involve parent education [17]. Outcome measures vary across studies and include target vs. non-target word production [18], expressive vocabulary test scores [19], general language test scores [20], spontaneous language sample measures such as MLU [21], parents' reports of communication and language skills [22], and changes in parents' language interactions [23] ${ }^{1)}$. The results from more than 25 studies trend in a positive direction, despite notable variability in children's performances within and across studies using similar measures [5,24].
The MacArthur-Bates Communicative Development Inventory (MB-CDI [2]), a standardized language assessment, is frequently included in treatment research for LTs as an identification and outcome variable. The primary goal of this measure is to evaluate young children's receptive and expressive vocabularies, along with their early morphological and syntactic productions. Six published intervention studies for LTs, as well as the present study, have used the MB-CDI (or its predecessor, the MacArthur Communicative Development Inventory (M-CDI [25])), to quantify post-treatment expressive vocabulary growth. The majority of these studies employed parent-training approaches that emphasized direct language stimulation techniques and are discussed below. The others share a similar theoretical background to the present investigation: statistical learning. These intervention studies will be discussed at the end of the next section.

Three of the four parent-training based LT intervention studies that measured expressive vocabulary outcomes via the MB-CDI [2] or M-CDI [25] utilized an intervention vs control group method. Girolametto et al. [18] was the only of these studies that resulted in significant group differences, with the experimental group producing nearly 3 times as many words by the end of the treatment. Girolametto et al. [26] and Roberts and Kaiser [19] reported similar growth in the expressive vocabularies of their experimental groups, but these improvements were not significantly different from those of the control groups. It is unclear why these two studies were not as successful as Girolametto et al. [18]. The number of sessions does not appear to be a primary factor, as both of Girolametto et al.'s investigations used the same number of sessions and Roberts and Kaiser [19] consisted of 3 times as many sessions. The pre-experimental size of the LTs' expressive vocabularies may have had an effect. Despite similar ages, the LTs in the study with significant group differences [18] began with expressive vocabularies that were half and one sixth of those in Girolametto et al. [26] and Roberts and Kaiser [19], respectively.

Cunningham et al. [22], the fourth parent-training based LT intervention study that utilized the MB-CDI, did not include a control group. Unlike the others, however, the participants' expressive vocabularies were measured at three time points: pre and post treatment, and later at a 6-12 week follow-up. Statistically significant differences were reported before and at

\footnotetext{
1) The studies listed here are exemplars of the types of outcome measures indicated, not an exhaustive list. Also, many of these and other intervention studies include multiple outcome measures.
} 
the end of the treatment (average increase of 2.4 times more words) and at the end of the treatment and 12 weeks later (average increase of 1.5 times more words). Due to the lack of a control group, it is unclear if these differences were the result of the intervention or maturation.

\section{Statistical learning and shape bias}

Statistical learning is a form of implicit learning in which individuals detect and use regularities within complex input to improve their knowledge and skills, primarily by providing evidence to make predications and reduce uncertainty [27]. It has been observed across language domains in typically functioning infants, children, and adults [28-30]. Children with language learning disorders, including specific language impairment and autism, have been shown to have poorer statistical learning skills than their peers with typical language development [31-33].

Lexical principles are a subcategory of statistical learning that help children become effective and efficient word learners $[34,35]$. The purpose of lexical principles is to reduce the number of potential meanings of novel words and guide generalization of their labels to other referents. They include the whole-object assumption, taxonomic assumption, mutualexclusivity assumption, and shape bias. While word learning is a complex/multifaceted process, the current study addresses the principle of shape bias because of its strong connections to rapid increases in young children's expressive vocabularies. Shape bias is built on the propensity of the names of concrete objects, specifically count nouns, to generalize to other objects that have the same shape, as opposed to other shared features such as color, texture, or pattern [36]. This allows children to generalize the object's name to other referents without repeated exposures and quickly build a categorical representation of the word. For example, a child who first learns the word plate in connection to the round, red, plastic dish she eats off of is more likely to generalize the name "plate" to other round objects than to things that are red or made of plastic. Like all lexical principles, shape bias provides an initial hypothesis that can be confirmed or rejected with additional experience. Children typically develop shape bias around two-years of age [37], when their vocabularies are dominated by count nouns [38].

Smith, Samuelson, and colleagues directly examined the potential connection between shape bias and expressive vocabulary development by teaching shape bias to children younger than 24 months old. Smith et al. [1] enrolled eight 17-month-old children in an 8-week intervention. Shape bias was targeted through focused stimulation-based play that included similarly shaped objects identified with the same spoken label and a differently shaped, unnamed object matched to the others by color and texture. By the final session, the children receiving the intervention outperformed age matched controls on shape bias assessment tasks and expressive vocabulary measures ( $256 \%$ increase vs. $78 \%$ increase). The intervention group also generalized shape bias learning to a) previously unseen objects with the same shapes as those in the intervention and b) novel objects with the same shape as unfamiliar objects and labels that were not part of the intervention. In another study, Samuelson [39] examined the expressive vocabulary development of 15- to 21-month-olds following exposure to novel nouns in one of three conditions a) nouns that are identifiable by shape (i.e., count nouns), b) shapeless, material-based nouns (i.e., mass nouns), and c) no target nouns. As with Smith et al. [1], the children with increased exposure to shape bias contexts performed better than those who did not. The positive increases in these children's expressive vocabularies were observed at the end of the intervention and one month later.

Despite the evidence that increased exposure to shape bias contexts can improve the vocabularies of children with typical development, there is limited research on the shape bias skills of LTs or children with other language impairments. Jones [40] examined the shape bias abilities of LTs and age-matched peers by asking them to match novel-named, unfamiliar objects to one of three other objects that were similar in either shape, color, or texture. The LTs did not demonstrate the shape bias; in fact, they only selected objects that matched in texture at above chance levels. Their peers, on the other hand, demonstrated shape bias selected by choosing only same shaped objects at above chance levels. Using similar procedures, Collison et al. [38] tested the effects of shape bias on noun learning in children with typical language development and children with SLI. As predicted, the children with SLI, like LTs, do not show shape bias as strongly as their age matched peers.

Alt and colleagues published two statistical learning-based studies designed to increase the expressive vocabularies of LTs $[6,7]$. Although neither addressed shape bias specifically, they are included here to demonstrate the potential for this category of intervention. Alt et al. [7] provided primarily clinician-directed input during 14-20 sessions to 4 LTs. Two steps were taken to maximize the potential for statistical learning: a) 
each session included at least 64 productions each of three or more target words and $b$ ) each word was presented in at least five different activities, with different clinicians, in a variety of utterance positions. By the end of the treatment, the participants had demonstrated improvements in the number of target words produced, and their expressive vocabulary raw and percentile scores on the MB-CDI [2]. Alt et al. [6] replicated these findings, as well as demonstrated significant vocabulary growth in experimental vs. delayed-treatment control group at the end of treatment and 4-6 weeks later.

\section{Present study}

Stokes et al. [41] proposed that LTs engage in a period of extended statistical learning, in which they are slower to extract and exploit regularities in speech input to improve their lexical abilities. This theory resulted from cross-linguistic examinations of the neighborhood densities and use of frequent words by two-year-olds with varying expressive vocabulary sizes [42]. The present study is an exploratory investigation examining the effectiveness of statistical learning-based interventions on the expressive vocabularies of LTs. As a pilot study, it is small in scale and designed to assess methodological efficiency and feasibility in order to guide and support future investigations [43-45]. The specific goals of this study are to acquire information about implementing Smith et al.'s [1] procedures with LTs and examine the efficacy potential of the intervention.

A number of a priori decisions were made in order to address these goals and complete the study in an economical, yet effective, manner. The most significant of these were to a) provide the same treatment to all of the participants, what El- dridge et al. [43] refer to as a non-randomized pilot study, and b) utilize a single-subject design (i.e., no control group), as has occurred in other intervention studies of LTs $[7,22,46]$. As a result, any positive changes in the LTs expressive vocabulary growth cannot be attributed to the intervention exclusively; however, comparing these children's development with those from Smith et al's [1] investigation and other interventions for LTs may provide sufficient information to guide additional research on the intervention.

\section{METHODS}

\section{Participants}

The 8 participants were seven males and one female between the ages of 23 and 34 months living in northwest Ohio. They were all identified by their parents as being Caucasian and two were reported to be Hispanic. All of the participants were monolingual English speakers, although one child reportedly had limited exposure to Spanish. Another participant was reported to have childhood apraxia of speech, although significant speech errors were not noted during his sessions. No other developmental delays or difficulties were reported. Maternal and paternal education ranged from high school diploma to doctorate degrees. At the time of the first intervention session, all of the participants had expressive vocabularies at or below the 10th percentile on the words and sentences form of the MB-CDI [2]. Table 1 presents each participants' age and pretest expressive vocabulary size.

The investigation was approved by the Bowling Green State University institutional review board. Recruitment began by contacting local child care centers and discussing the study

Table 1. Expressive vocabulary counts and shape-bias generalization scores, organized by age

\begin{tabular}{|c|c|c|c|c|c|c|c|c|}
\hline & \multicolumn{8}{|c|}{ Participant Number } \\
\hline & 1 & 2 & 3 & 4 & 5 & 6 & 7 & 8 \\
\hline Age at first session (month) & 23 & 24 & 25 & 25 & 27 & 28 & 33 & 34 \\
\hline \multicolumn{9}{|l|}{ Expressive vocabulary ${ }^{\mathrm{a}}$} \\
\hline Pretest & 25 & 79 & 29 & 124 & 89 & 58 & 232 & 198 \\
\hline Posttest & 87 & 147 & 53 & 230 & 131 & 124 & 366 & 317 \\
\hline Total words added & 62 & 68 & 24 & 106 & 42 & 66 & 134 & 119 \\
\hline Percentage increase & $248 \%$ & $86 \%$ & $83 \%$ & $85 \%$ & $47 \%$ & $114 \%$ & $58 \%$ & $60 \%$ \\
\hline \multicolumn{9}{|l|}{ Generalization scores } \\
\hline First-Order & $3 / 4$ & $4 / 4$ & $3 / 4$ & $4 / 4$ & $2 / 4$ & $4 / 4$ & $2 / 4$ & $2 / 4$ \\
\hline Higher-Order & $2 / 4$ & $2 / 4$ & $3 / 4$ & $3 / 4$ & $2 / 4$ & $3 / 4$ & $3 / 4$ & $2 / 4$ \\
\hline
\end{tabular}

${ }^{\mathrm{a}}$ Total number of words as measured by the MacArthur-Bates Communicative Development Inventory [2]. 
with their directors. Upon approval, copies of the information and consent forms were dispersed to families by the child care providers. Families interested in the study contacted the research team directly or indirectly through the child care staff.

\section{Stimuli and procedures}

The protocol of Smith et al.s [1] shape bias intervention was followed as closely as possible, with the exception of a few intentional differences that are described below. The examiner met individually with each child for 25-30-minute sessions, once per week, for eight weeks. During each session, the child and examiner played with the four sets of novel objects displayed in Figure 1. The novel objects were all handmade 3D cuts of Styrofoam that were covered by cloth, paper, paint, or another material. Each set included two objects that were the same shape but different in color and texture. The third object was a different shape than the others, but the same color as one of the shape-matched pair and the same texture as the other. The shaped-matched objects were labeled with the same nonsense word. The labels used across the four sets were "dax," "lug," "wif," and "zup." No label was given for the object in each set that did not match the others by shape. Instead, it was referred to as "not a __." The examiner and child played with each set of objects for 5 minutes. During that time, the examiner was to produce the target word's label at least 10 times and the phrase "not a _ _ at least 5 times. The child was not requested or required to use any of the target labels during the intervention sessions.

On the eighth week, each of the children's abilities to generalize the novel names to other objects of the same shape was tested. As shown in Figures 2 and 3, each of these eight trials included presentation of a target novel object and its label, and three other objects that matched the target by either shape, color, or texture. Four of the trials consisted of a target object and word from the intervention and three novel objects that were not seen in the previous sessions. The examiner

\section{$\operatorname{Dax}$}

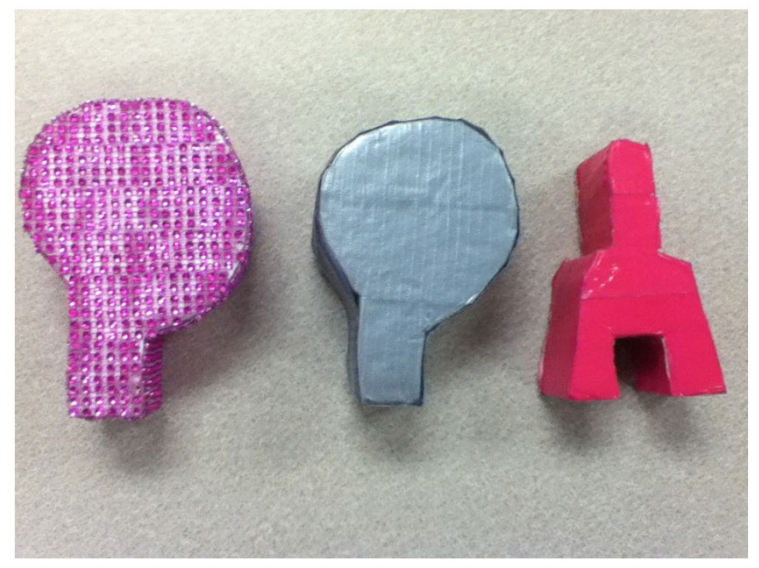

Wif

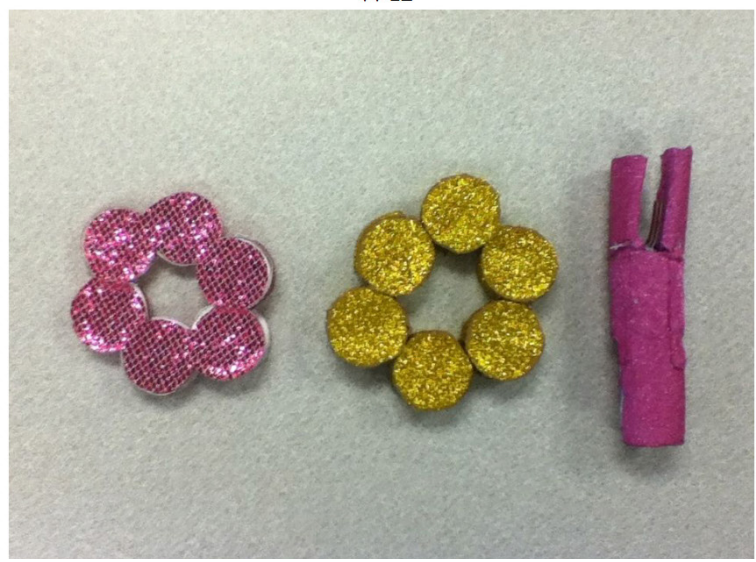

Lug

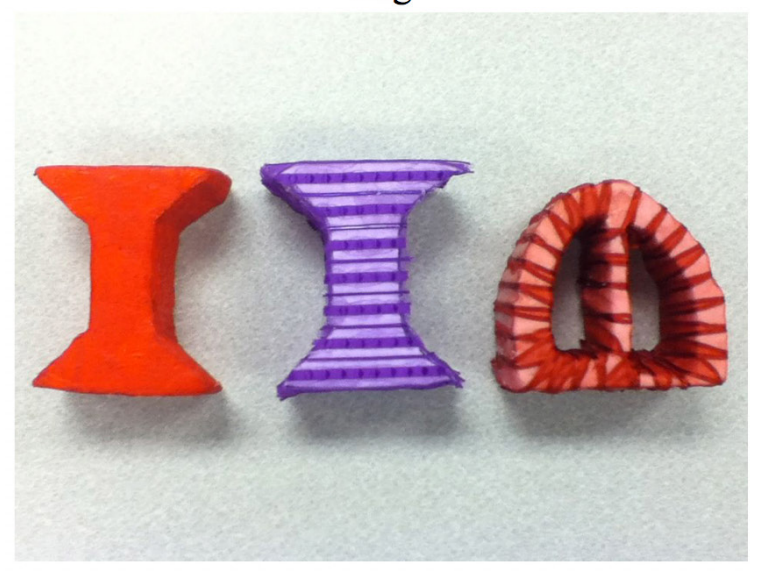

Zup

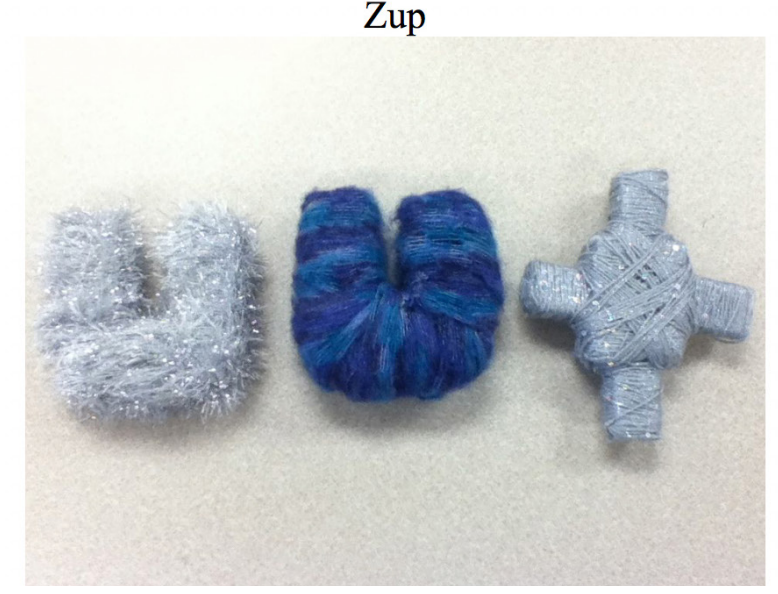

Figure 1. The Four Sets of Novel Objects and Associated Nonsense Labels Used in Training. 


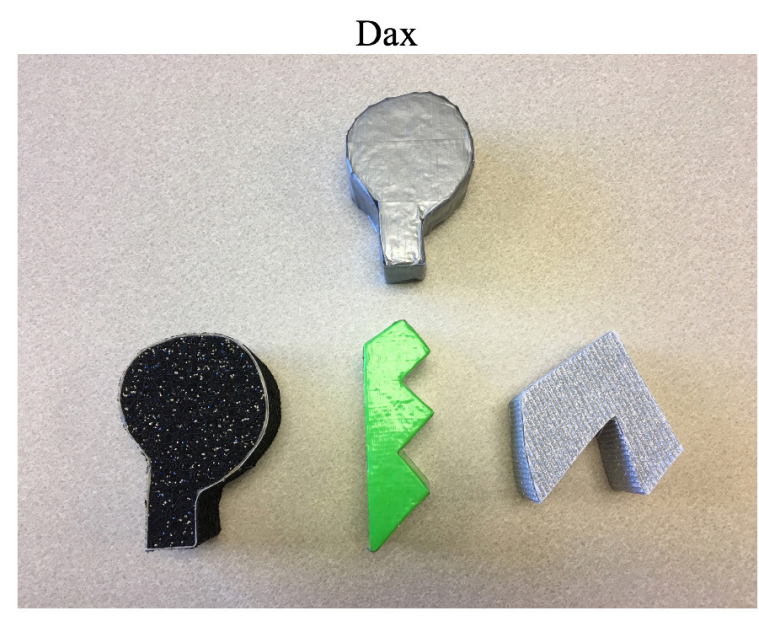

Wif

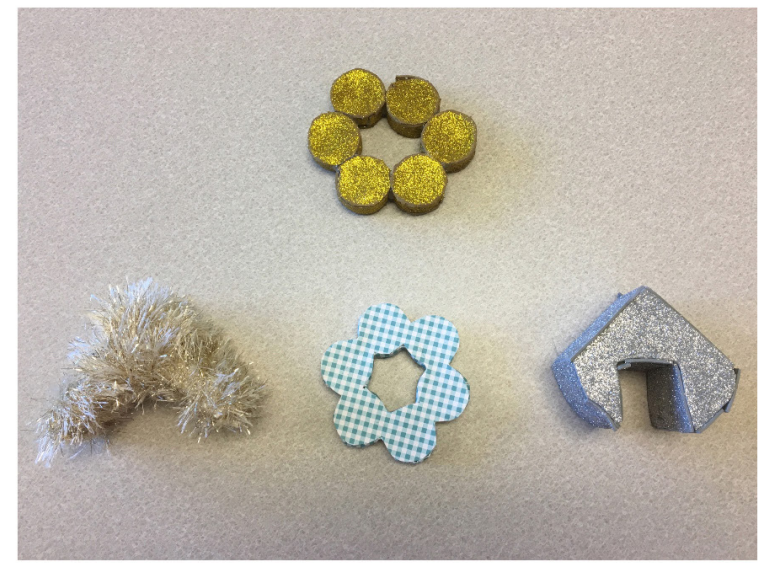

Figure 2. First-Order Generalization Objects and Labels.

showed the target object and said, "This is a __." then pointed to the other objects and said, "Where's a __? Get me a __." Smith et al. [1] identified these as first-order generalizations because they demonstrate extensions from objects that had previously been labeled. The other four trials consisted of higher-order generalizations because they assessed the application of shape bias to unfamiliar objects and labels that were not part of the intervention. These trials were the children's first exposure to the shapes and labels of the target objects. Each child's first consistent point to an object was scored as their response. It is noted that some of the children first pointed to all of the objects before settling on one. These generalization measures were all in the domain of receptive language skills. To measure the children's expressive vocabulary growth, the MB-CDI [2] was given to their parents a second time, at Week 7, and returned at the final session. All of the words that were identified as having been produced at pretest were credited on the posttest administration.

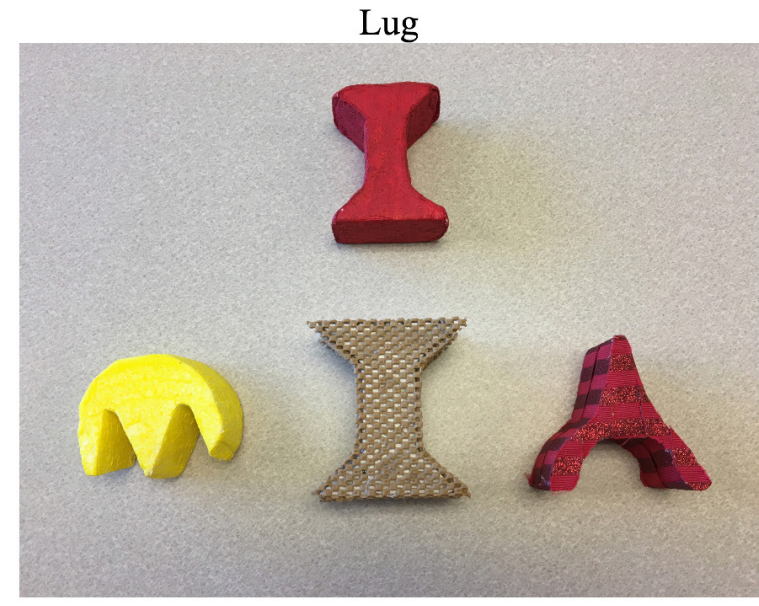

Zup

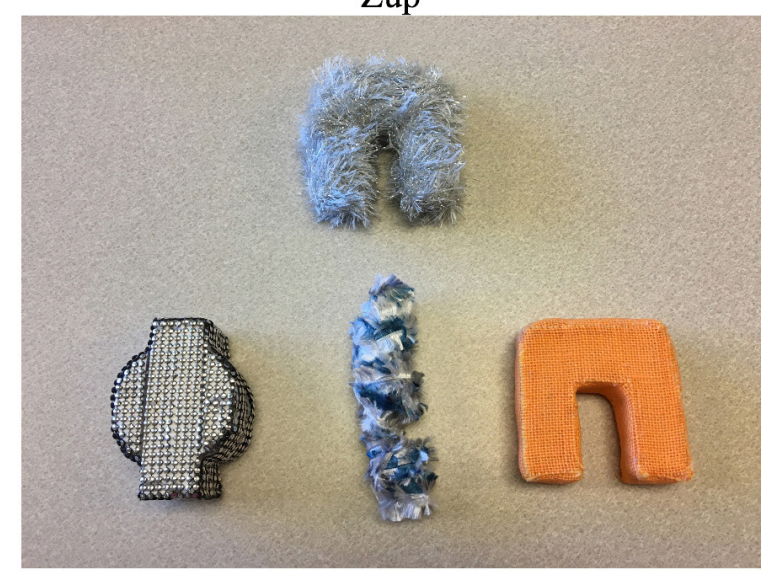

The following intentional changes were made to Smith et al.s [1] protocol. Although Smith et al. did not report where the sessions occurred, parents/guardians took part in each session. In the present study, the children were seen in either their home, child-care center, or the university clinic. Parents/ guardians were invited to participate in the sessions, but were not required to. The hand-made objects used in the present study matched those used by Smith et al. for size and some of the shapes, as not all of shapes used by Smith et al. were identified. The textures of the objects differed between the two studies, with Smith et al. using different building materials (e.g., wood, sponge, and plastic) and the present study applying coverings with different textures to Styrofoam bases. Smith et al. did not specify the type of play used in the sessions. Play in the present study incorporated techniques from naturalistic, child-centered language interventions, including following the child's lead and narrating the play (e.g., "The zup is driving the truck." and "That hit me. It's not a zup.") [47]. Fi- 


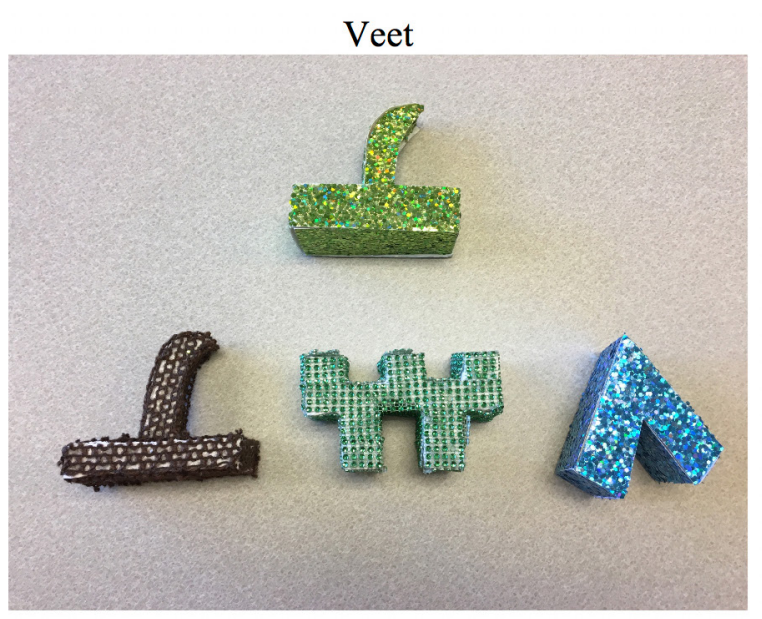

Nim

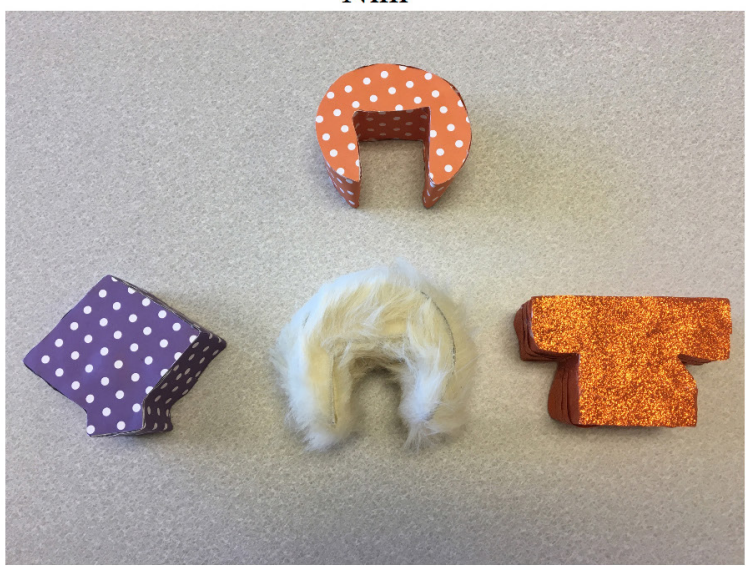

Figure 3. Higher-Order Generalization Objects and Labels.

nally, Smith et al.'s [1] intervention lasted a total of 9 weeks, with the last two sessions consisting of the first- and higherorder generalization tests separately. These tasks were combined into a single session in the present study in order to save the participants and their families from needing to attend two very short final sessions.

\section{RESULTS}

Each participant's expressive vocabulary size, as measured by the MB-CDI [2], and generalization test score is shown in Table 1. Over the eight-week period, the LTs added 24-134 words to their expressive vocabularies, with an average increase of 78 words. Because each child started with a different number of words in their expressive vocabularies, the percent of new words added between the two administrations of the MB-CDI was considered for comparative purposes. The degree to which the participants' expressive vocabularies increased
Teema

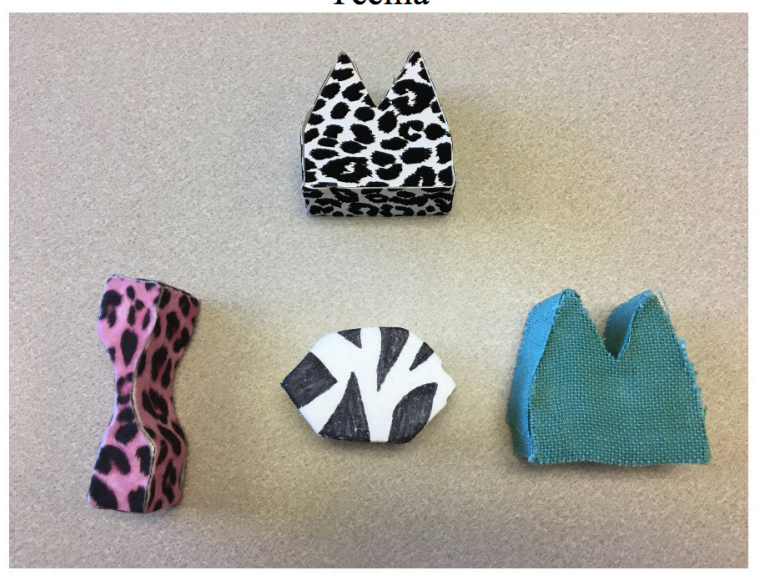

Gazzer

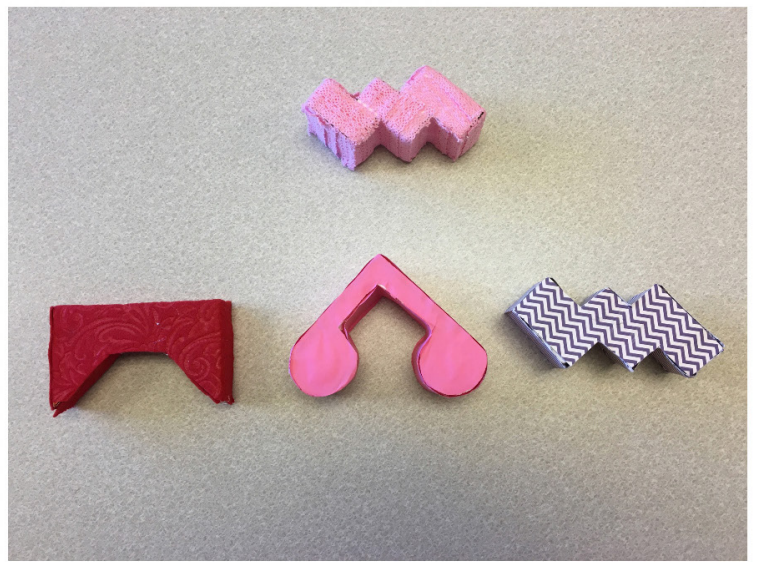

ranged from $47 \%$ to $248 \%$, with three performance groups emerging from the data. Participants 1 and 6 demonstrated the highest increases, $248 \%$ and $114 \%$, respectively. Increases of approximately $85 \%$ were observed for participants 2,3 , and 4. Finally, the smallest increases were displayed by participants 5, 7, and 8, who improved their expressive vocabularies by $47-60 \%$. Visual inspection of the data are inconclusive regarding the potential connections between the posttest results and age or pretest vocabulary size.

The two generalization probes measured the participants' abilities to extend novel labels to objects matched by shape. On the first-order generalization trials, the LTs applied the trained names to new objects with the same shape with 50$100 \%$ accuracy (i.e., on two to four of the trials). Their performances on the higher-order generalization trials, in which they applied new novel names to previously unseen objects with the same shape, ranged from $50-75 \%$ accuracy.

To examine the generalization trials' results further, the 
odds of responding correctly across each set by chance was calculated by using the binomial probability formula: $\mathrm{b}(x ; n$, $P)={ }_{\mathrm{n}} \mathrm{C}_{\mathrm{x}} \times \mathrm{P}^{\mathrm{x}} \times(1-\mathrm{P})^{\mathrm{n}-\mathrm{x}}$, where $x=$ the number of correct trials, $n=$ the number of total trials, $P=$ the probability of success on any single trial, $\mathrm{C}=$ the number of combinations that could yield the successful response rate. The odds of responding correctly by chance to 2,3 , and 4 items within each set of trials was $0.29,0.10$, and 0.01 , respectively. As a result, performances of 75 and $100 \%$ accuracy were interpreted as demonstrations of successful first- and higher-order generalizations because of their low probability of occurring by chance. As shown in Table 1, three of the participants were successful for both types of generalization (participants 3, 4, and 6), three on one type of generalization (participants 1,2, and 7), and two on neither (participants 5 and 8). There did not appear to be a direct relationship between generalization success and expressive vocabulary growth for children with middle to high increases. The two children who were not successful with either generalization task, however, had the smallest increases in expressive vocabulary.

\section{DISCUSSION}

\section{Implementing Smith et al.'s [1] Protocol with LTs}

The first goal of this study was to acquire information about applying the procedures used by Smith et al. [1] to LTs. Forty unique 3D objects were created from Styrofoam and covered by different materials. Although this process took a great deal of time and planning, it was critical to ensure that the objects' shapes, patterns, and colors matched their intentions and did not overlap with other objects. The children were generally interested in the activity and it was not difficult to meet the five minutes of play with each set of objects. In the few instances where a child showed disinterest, the examiner followed the child's lead to change the play and then incorporated the objects back into the activity.

Each child's sessions occurred at either their home, childcare center, or the university clinic. Parents/guardians of the children seen in their home or the clinic were invited to sit in on the intervention sessions. They were encouraged to participate in the play during the first few sessions to help the child feel comfortable in the experience. Most did not regularly participate in the subsequent sessions, other than occasional interactions when the child directly addressed them. None of the parents/guardians made overt attempts during the sessions to directly teach the nonsense words or interfered in any notable way. Overall, the inclusion of parents/guardians in the sessions did not appear to either interfere or be necessary for successful implementation of the protocol.

As noted earlier, the experimenter attempted to produce each target label 10 or more times per session and the "not a _" phrase at least 5 times. The requirement for the labels was easily met within each 5 -minute period, but the "not a _" phrases were typically used between 2 and 5 times per period. A second examiner attended multiple sessions for the first two participants to observe and provide feedback, but did not participate in subsequent sessions once it was determined that the clinician did not need this support. The primary examiner's variability in exposure, however, suggests repeated evaluation by either the primary or secondary examiner to ensure that the prescribed number of exposures is achieved and consistent across participants.

\section{Impacts of the intervention}

After completing the intervention, many of the LTs' parents reported that they noticed positive differences in their child's expressive language, both in the number of words used and the frequency of verbal attempts. This may have been a result of the intervention or a form of bias, because they were not blinded to the sessions or their purpose. All of the children increased their expressive vocabularies by the end of the eighth week, as expected by both the intervention and the expressive vocabulary development of 2-year-olds with typical development over a 2-month period [2]. Six of the eight participants demonstrated successful generalization of shape bias to novel objects and/or novel objects paired with novel words. Taken together, these results are in a positive direction, but do not indicate if the LTs' progress was directly due to the intervention or other maturational factors. The present research cannot make this determination due to the lack of a control group. Comparing the results of this study with those of Smith et al. [1] and other interventions for LTs, however, may help to determine if they were strong enough to lead to a larger-scale investigation.

The children in Smith et al.'s [1] intervention and control groups increased their expressive vocabularies by averages of 51 and 24 words, respectively. All but one of the LTs in the present study added as many or more words as Smith et al's intervention group. The remaining LT's increase was between that of Smith et al.'s intervention and control groups. It is noted that the children in the present study were older and often began with larger expressive vocabularies than those 
from Smith et al. To compensate for this, a comparison between the percentages of noun increase was conducted. On average, the LTs in this study showed an increase of $116 \%$ in their expressive noun vocabularies, which is between Smith et al's experimental group and control groups $(256 \%$ and $78 \%$, respectively). Only one of the LTs in the present study, LT 1, increased nouns at a rate similar to or above the mean of Smith et al's experimental group. LT 3 had an increase between their experimental and control groups, while the others were at or below Smith et al.'s control group. Overall, the shape bias intervention was not as successful for the LTs in the present study as it was for the children with typical language development from Smith et al. The degree to which this is due to differences with how LTs and their peers acquire language, the participants' age differences, variations in the administration of the protocol, or a combination of these and other factors is unknown.

Comparing the results of the present study with those of other LT intervention research may shed further light on the success of applying the shape bias intervention to this population. A review of the LT intervention literature revealed six studies whose outcome variables also included the MB-CDI [2]. These studies used different intervention approaches than the present study and each other, and their treatments were longer and/or more intensive than the present research. The participants in three of these studies $[6,7,18]$ showed two to three times greater increases in their expressive vocabularies than those in the present study. LTs in the other two studies increased their expressive vocabularies by as many or fewer words than those in the present research $[21,26]$.

The results of the present study are mixed. On the one hand, the increases in the LTs expressive vocabularies were below those of a) younger children with typical language development receiving the same intervention and b) LTs receiving some other forms of intervention. On the other hand, their vocabulary increases were on par or above those of LTs receiving different interventions. One common trend across each of these studies, and the majority of LT intervention research, is a large amount of variability between participants. The complexities within and between the results of these studies suggest that more research on interventions for LTs, including shape bias and other forms of statistical learning, is warranted.

\section{Future directions}

Two important limitations of this pilot study were the lack of control for maturational effects and limited information about the participants' language skills before and after the intervention. To address these concerns, a randomized, mixed-design (within and between groups) study is planned. The first session for each participant will consist of language testing, including a generalized language measure (e.g., the ReceptiveExpressive Emergent Language Test, 3rd edition [48]), the MB-CDI [2], and a measure of shape bias ability (the generalization tasks from the present study). Participants identified as LTs will be randomly placed into an earlier- or later-treatment group. The earlier-treatment group will receive the intervention during the subsequent seven weeks, while the later-treatment group will be held in control. Both groups will receive follow-up testing in week 9 that include the MB-CDI and shape bias task. The sessions will be increased to twice per week and the number of exposures to each target novel word will be doubled. Treatment status for the groups will switch for weeks 10-16, with the earlier-treatment group in control and the later-treatment group receiving the intervention. Final testing, repeating the same two measures, will conducted at week 17. Between-group comparisons of expressive vocabulary change at weeks 9 and 17 will evaluate treatment vs. maturation and treatment vs. maintenance, respectively. Within-participant comparisons will explore the effects of the treatment within each group and for each individual.

There are a number of directions that additional investigations of this nature could take. The effects of dosage, for example, could be examined by providing varying time and density of the intervention [6]. Additional statistical learningbased aspects of lexical development, such as fast mapping and neighborhood density, could be added to the protocol, especially because they are also problematic for LTs $[42,49]$ (see [50] for counter evidence). Finally, the shape bias treatment could be directly compared to more traditional vocabulary-teaching-based approaches, and/or a hybrid model.

\section{Summary}

Smith et. al.'s [1] shape bias intervention was successfully carried out with a group of LTs. Lessons in implementing the protocol for future research include careful detail in developing the stimuli, a perceived lack of effect related to including parents/guardians in the sessions, and improving the intervention fidelity. All eight LTs increased their expressive vocabularies after completing the intervention, although maturational effects could not be ruled out. The LTs vocabulary growth was variable, but commensurate with the results of some, but not all, other LT intervention studies using the 
same outcome measure. These findings support the development of larger-scale investigations into the shape bias intervention's potential for improving the expressive vocabulary skills of LTs, but does not warrant its adoption into clinical practice at the present time.

\section{REFERENCES}

1. Smith LB, Jones SJ, Landau B, Gershkoff-Stowe L, Samuelson L. Object name learning provides on the job training for attention. Psychological Science. 2002;13:13-19.

2. Fenson L, Marchman VA, Thal DJ, Dale PS, Reznick JS, \& Bates E. MacArthur-Bates communicative development inventories: User's guide and technical manual (2nd ed.). Baltimore, MD: Brookes; 2007.

3. Gleason J, Ratner N. The development of language (7th ed.). Boston: Pearson Education, Inc.; 2009.

4. Desmarais C, Sylvestre A, Meyer F, Bairati I, Rouleau N. Systematic review of the literature on characteristics of late-talking toddlers. International Journal of Language \& Communication Disorders. 2008;43:361-389.

5. Cable AL, Domsch C. Systematic review of the literature on the treatment of children with late language emergence. International Journal of Language \& Communication Disorders. 2011;46:138154.

6. Alt M, Mettler HM, Erikson JA, Figueroa CR, Etters-Thomas SE, Arizmendi GD, Oglivie T. Exploring input parameters in an expressive vocabulary treatment with late talkers. Journal of Speech, Language, and Hearing Research. 2020;63:216-233.

7. Alt M, Meyers C, Oglivie T, Nicholas K, Arizmendi G. Cross-situational statistically based word learning intervention for late-talking toddlers. Journal of Communication Disorders. 2014;52:201220.

8. Paul R. Profiles of toddlers with slow expressive language development. Topics in Language Disorders. 1991;11:1-14.

9. Zubrick SR, Taylor CL, Rice ML, Slegers DW. Late language emergence at 24 months: an epidemiological study of prevalence, predictors, and covariates. Journal of Speech, Language, and Hearing Research. 2007;50:1562-1592.

10. Bavin EL, Bretherton L. The early language in Victoria study: late talkers, predictors, and outcomes. In Rescorla, LA, Dale, PS (Eds.), late talkers: language development, interventions, and outcomes (3-21). Maryland: Paul H. Brookes Publishing Co.; 2013.

11. Rescorla L. Age 17 language and reading outcomes in late-talking toddlers: support for a dimensional perspective on language delay. Journal of Speech, Language, and Hearing Research. 2009;52: 16-30.

12. Rescorla L, Turner HL. Morphology and syntax in late talkers at age 5. Journal of Speech, Language, and Hearing Research. 2015; 58:434-444.

13. Taylor CL, Zubrick SR, Rice ML. Population and public health perspectives on late language emergence at 24 months as a risk indicator for language impairment at 7 years. In Rescorla, LA, Dale, PS (Eds.), late talkers: language development, interventions, and outcomes (23-40). Maryland: Paul H. Brookes Publishing Co.; 2013.

14. Fisher EL. A systematic review and meta-analysis of predictors of expressive-language outcomes among late talkers. Journal of Speech, Language, and Hearing Research. 2017;60:2935-2948.

15. Thal D, Tobias S, Morrison D. Language and gesture in late talkers: a 1-year follow-up. Journal of Speech, Language, and Hearing Research. 1991;34:604-612.

16. Finestack LH, Fey ME. Evidence-based language intervention approaches for young late talkers. In Rescorla, LA, Dale, PS (Eds.), late talkers: language development, interventions, and outcomes (283-302). Maryland: Paul H. Brookes Publishing Co.; 2013.

17. Girolametto L, Weitzman E, Earle C. From words to early sentences: Parent-implemented intervention for late-talking toddlers. In Rescorla, LA, Dale, PS (Eds.), late talkers: language development, interventions, and outcomes (261-282). Maryland: Paul H. Brookes Publishing Co.; 2013.

18. Girolametto L, Pearce PS, Weitzman E. Interactive focused stimulation for toddlers with expressive vocabulary delays. Journal of Speech and Hearing Research. 1996;39:1274-1283.

19. Roberts MY, Kaiser AP. Early intervention for toddlers with language delays: a randomized controlled trail. Pediatrics. 2015;235: 686-693.

20. Buschmann A, Bettina M, Hasselhorn M, Pietz J. Long-term effects of a parent-based language intervention on language outcomes and working memory for late-talking toddlers. Journal of Early Intervention. 2015;37:175-189.

21. Robertson SB, Ellis Weismer S. Effects of treatment on linguistic and social skills in toddlers with delayed language development. Journal of Speech, Language, and Hearing Research. 1999;42:1231248.

22. Cunningham BJ, Kwok E, Earle C, Oram Cardy J. Exploring participation and impairment-based outcomes for Target Word ${ }^{\mathrm{TM}}$ : a parent implemented intervention for preschoolers identified as lateto-talk. Child Language, Teaching, and Therapy. 2019;35:145-164.

23. Kruythoff-Broekman A, Wiefferink C, Rieffe C, Uilenburg N. Parent-implemented early language intervention programme for late talkers: Parental communicative behaviour change and child language outcomes at 3 and 4 years of age. International Journal of Language \& Communication Disorders. 2019;54:451-464.

24. DeVeney SL, Hagaman JL, Bjornsen AL. Parent-implemented versus clinician-directed interventions for late-talking toddlers: a systematic review of the literature. Communication Disorders Quarterly. 2017;39:293-302.

25. Fenson L, Reznick S, Thal D, Bates E, Hartung J, Pethick S, Reilly J. The MacArthur Communicative Developmental Inventory. San Diego, CA: Singular Publishing Group, Inc.; 1993.

26. Girolametto L, Pearce K, Weitzman E. The effects of focused stimulation for promoting vocabulary in young children with delays: a pilot study. The Division for Children's Communication Development. 1996;17:39-49. 
27. Saffran JR. Statistical language learning in infancy. Child Development Perspectives. 2020;14:49-54.

28. Palmer SD, Hutson J, Mattys SL. Statistical learning for speech segmentation: age-related changes and underlying mechanisms. Psychology and Aging. 2018;33:1035-1044.

29. Plante E, Gomez RL. Learning without trying: the clinical relevance of statistical learning. Language, Speech, and Hearing Services in Schools. 2018;49:710-722.

30. Saffran JR, Aslin RN, Newport EL. Statistical learning by 8-monthold infants. Science. 1996;274:1926-1928.

31. Alt M. How statistical learning relates to speech-language pathology [Special issue]. Language, Speech, and Hearing Services in Schools, 11(3s); 2018.

32. Evans JL, Saffran JR, Robe-Torres K. Statistical learning in children with specific-language impairment. Journal of Speech, Language, and Hearing Research. 2009;52:321-335.

33. Lammertink I, Boersma P, Wijnen F, Rispens J. Statistical learning in specific language impairment: a meta-analysis. Journal of Speech, Language, and Hearing Research. 2017;60:3474-3486.

34. Golinkhoff RM, Hirsh-Pasek K, Bloom L, Smith LB, Woodward AL, Akhtar N, Tomasello M, Hollich G. Becoming a word learner: a debate on lexical acquisition. Oxford: Oxford University Press; 2000.

35. Markman EM. Constraints children place on word meanings. Cognitive Science. 1990;14:57-77.

36. Smith LB. An associative crane. In becoming a word learner: a debate on lexical acquisition. Oxford: Oxford University Press; 2000.

37. Hupp JM. Development of the shape bias during the second year. The Journal of Genetic Psychology. 2015;176:82-92.

38. Collison BA, Grela B, Spaulding T, Rueckl JG, Magnuson JS. Individual differences in the shape bias in preschool children with specific language impairment and typical language development: theoretical and clinical implications. Developmental Science. 2014;17:1-16.
39. Samuelson LK. Statistical regularities in vocabulary guide language acquisition in connectionist models and 15-20-montholds. Developmental Psychology. 2002;38:1016-1037.

40. Jones SS. Late talkers show no shape bias in novel name extension task. Developmental Science. 2003;6:477-483.

41. Stokes SF, Kern S. Dos Santos. Extended statistical learning as an account for slow vocabulary growth. Journal of Child Language. 2012;39:105-129.

42. Stokes SF. Neighborhood density and word frequency predict vocabulary size in toddlers. Journal of Speech, Language, and Hearing Research. 2010;53:670-683.

43. Eldridge SM, Chan CL, Campbell MJ, Bond CM, Hopewell S, Thabane L, Lancaster GA. CONSORT 2010 statement: extension to randomized pilot and feasibility trials. BMJ. 2016;355:1-28.

44. Kaur N, Figueiredo S, Bouchard V, Moriello C, Mayo N. Where have all the pilot studies gone? A follow-up on 30 years of pilot studies in Clinical Rehabilitation. Clinical Rehabilitation. 2017;31: 1238-1248.

45. Leon AC, Davis LL, Kraemer HC. The role and interpretation of pilot studies in clinical research. Journal of Psychiatric Research. 2011;45:626-629.

46. DeVeney SL, Cress CJ, Reid R. Comparison of two word learning techniques and the effect of neighborhood density for late talkers. Communication Disorders Quarterly. 2014;35:133-145.

47. Paul R, Norbury CF. Language disorders from infancy through adolescence (4th ed.). St. Louis, MO: Elsevier; 2012.

48. Bzoch KR, League R, Brown V. Receptive-expressive emergent language test, 3rd edition. Austin, Tx: Linguistems; 2003.

49. Ellis Weismer S, Venker CE, Evans JE, Jones Moyle M. Fast mapping in late talking toddlers. Applied Psycholinguistics. 2013;34: 69-89.

50. Jones SD, Brandt S. Neighborhood density and word production in delayed and advanced learners. Journal of Speech, Language, and Hearing Research. 2019;62:2847-2854. 